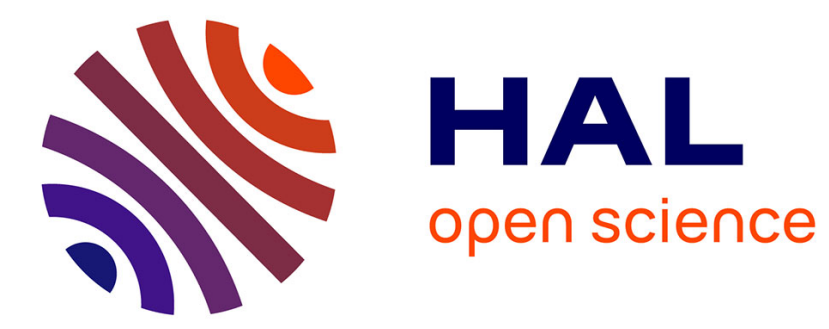

\title{
SOUDAGE D'ACIER Z 2 CN 18-10 PAR LASER CO2
}

\author{
B. Petesch, A. Sakout, M. Laurent, M. Robin
}

\section{To cite this version:}

B. Petesch, A. Sakout, M. Laurent, M. Robin. SOUDAGE D'ACIER Z 2 CN 18-10 PAR LASER CO2. Journal de Physique Colloques, 1987, 48 (C7), pp.C7-185-C7-186. 10.1051/jphyscol:1987735 . jpa-00227039

\section{HAL Id: jpa-00227039 https://hal.science/jpa-00227039}

Submitted on 1 Jan 1987

HAL is a multi-disciplinary open access archive for the deposit and dissemination of scientific research documents, whether they are published or not. The documents may come from teaching and research institutions in France or abroad, or from public or private research centers.
L'archive ouverte pluridisciplinaire $\mathbf{H A L}$, est destinée au dépôt et à la diffusion de documents scientifiques de niveau recherche, publiés ou non, émanant des établissements d'enseignement et de recherche français ou étrangers, des laboratoires publics ou privés. 
SOUDAGE D'ACIER Z $2 \mathrm{CN}$ 18-10 PAR LASER $\mathrm{CO}_{2}$

\author{
B. PETESCH, A. SAKOUT, M. LAURENT et M. ROBIN \\ CALFETMAT/IUT, 17, Rue de France, F-69100 Villeurbanne, France
}

Rèsumé : Le soudage d'un acier Z $2 \mathrm{CN} 18$ par faisceau laser a êté étudié. I'influence des principaux paramètres vitesse, puissance, focalisation a été examinée. Les moyens classlqques de caracțérisation des soudures ont étê utilisées : observations metallographịgues forme de la zone fondue, quantité de porosités et énergie absorbée. Le maximum de pénétration correspond au maximum d'énergie absorbée.

Abstract : - The LASER welding of $304 \mathrm{~L}$ steel has been studied. The welding parameters taken into account have been the welding speed, the power and the focusing of the laser beam. Classical weld characterisation means have been employed i.e. metallographic observations, weld shape, amount of porosities and input energy. The maximum of penetration as a function of focus point position corresponds to the maximum of input energy.

De nombreux paramètres sont à considêrer pour le soudage de matêriaux par faisceau laser ; parmi ces paramêtres la position de la tache focale par rapport à la surface, la puissance incidente et la vitesse de déplacement de la pièce sont particulièrement importantes. Nous présentons ici principalement 1 'influence de chacun de ces paramètres sur la morphologie de la zone fondue pour un acier austénitique $2 \quad 2$ CN $18-10$. Des lignes de fusion ont été effectuées avec deux lasers $\mathrm{CO} 2$ continus de puissance maximum $1000 \mathrm{~W}$ et $3500 \mathrm{~W}$ (CI 1000, CI 4000, CILAS). Les pièces ont étẻ rectifiées et soigneusement nettoyées dans un bain à $60^{\circ} \mathrm{C}$ de phosphate trisodique et silicate de soude, puis après rinçage, immergêes dans une solution d'acide nitrique à $10 \%$. A la suite d'essais préliminaires, le faisceau a été focalisé à l'aide d'une lentille de $127 \mathrm{~mm}$ de distance focale et la protection gazeuse a été assurée par de 1 'Hélium injecté par un tube incliné à $30^{\circ}$ par rapport à la surface de $1 a$ pièce. Pour examiner 1 'influence d'un des trois paramètres focalisation, puissance, vitesse, les deux autres ont été fixés et 1 'on a observé, la forme de la zone fondue, Ia quantité de porosités, I'énergie absorbẻe par la pièce et l'ênergie utile nếcessaire pour fondre un volume d'acier correspondant à la zone fondue. Sur la figuxe 1 est reportëe, pour une puissance de $2000 \mathrm{~W}$ et une vitesse de $3 \mathrm{~m} / \mathrm{mn}$, la pënétration en fonction de la focalisation. Pour cette puissance la vitesse de $3 \mathrm{~m} / \mathrm{mn}$ correspond à des cordons sains et profonds lorsque la pénétration est maximale. On peut constater sur cette figure que le maximum de pénétration a lieu lorsque la position de la tache focale est au voisinage de la surface et légèrement sous la surface (- $0,5 \mathrm{~mm})$. Il faut noter que pour obtenir une bonne pénétration il est nécessaire de contrôler avec précision la position focale, à quelques dixièmes de millimètres près. La figure 2 montre 1 'ézolution de la pénétration et de la largeur du cordon en fonction de la puissance pour une focalisation de $-0,5 \mathrm{~mm}$ et une vitesse de $3 \mathrm{~m} / \mathrm{mn}$. La pênétration croit beaucoup plus rapidement que la largeur, ceci correspond à des densités de puissance plus importantes à la surface de la pièce qui permettent de vaporiser plus rapidement l'alliage et ainsi de le creuser et d'établir un "trou de serrure" plus profond. L'intérêt de disposer de sources lasers multikilowatt apparait nettement. Cependant la courbe de pénêtration s'infléchit vers les fortes puissances, le plasma formé en surface devenant plus opaque et réflecteur.

La pénétration $P$ en fonction de $1 a$ vitesse $V$ suit approximativement une loi de la forme :

$$
P=a e^{-b V}
$$

où $a$ et $b$ sont des constantes pour une focalisation et une puissance donnée (voir tableau 1). Cette expression indique que lorsque la vitesse diminue la pénétration tend vers une limite, la pénétration augmente peu et le cordon s'élargit en surface. 
Il est intēressant de noter que l'énergie absorbée par la pièce est liëe à la forme de la zone fondue. Lorsqu'on fait varier la focalisation le maximum de pénétration correspond au maximum d'énergie absorbée. Pour une focalisation à 0,5 mm sous 1 a surface et une vitesse de $3 \mathrm{~m} / \mathrm{mn}$ pour des puissances comprises entre 800 et $3200 \mathrm{~W}$, 1 'énergie absorbée correspond approximativement à $55 \%$ de l'énergie incidente et 1 'énergie utile à $25 \%$.

FIGURE 1

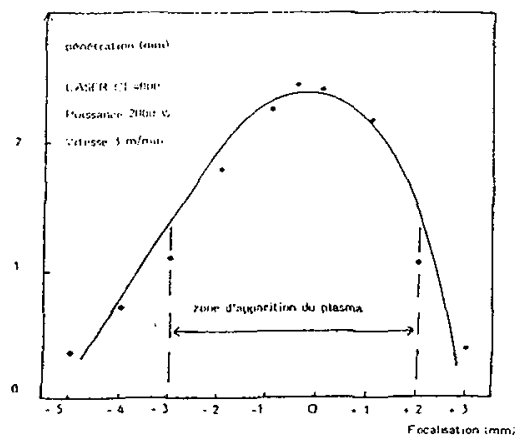

Influence de la focalisation sur la pénétration (laser CI 4000, puissance : $2000 \mathrm{~W}$, Vitesse: $3 \mathrm{~m} / \mathrm{mn}$ ).

Penetration as a function of focus point position (Power $2 \mathrm{~kW}$, speed : $3 \mathrm{~m} / \mathrm{mn}$, CI 4000 laser).
FIGURE 2

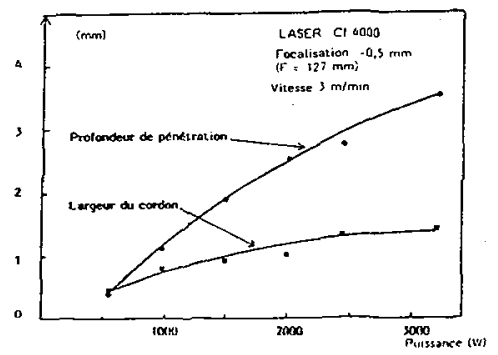

Influence de la puissance sur $1 a$ pénétration et la largeur du cordon (1aser CI 4000, focalisation : - 0,5 mm vitesse : $3 \mathrm{~m} / \mathrm{mn}$ ) Penetration and widtif as functions of power. (Focus point position : - $0,5 \mathrm{~mm}$, speed : $3 \mathrm{~m} / \mathrm{mn}$, CI 4000 CI 4000 Laser).

TABLEAU I

\begin{tabular}{|c|l|c|}
\hline Puissance incidente & $a(\mathrm{~mm})$ & $b(\mathrm{~mm} / \mathrm{m})$ \\
\hline 800 & 1,7 & 0,13 \\
2000 & 3,5 & 0,16 \\
3200 & 5 & 0,15 \\
\hline
\end{tabular}
Termes de l'expression $P=a e^{-b V}$ pour plusieurs puissances donnant
en fonction de la vitesse pour une focalisation de $-0,5 \mathrm{~mm}$ (CI 4000 ). Termes de 1 'expression $P=a e^{-b V}$ pour plusieurs puissances donnant la pénétration
en fonction de la vitesse pour une focalisation de $-0,5$ mm (CI 4000 ).

Terms of $P=a e^{-b V}$ (focus point position : - 0,5 mm, CI 4000 Laser)

En conclusion, le laser continu $\mathrm{CO} 2$ constitue un moyen de soudage donnant pour un acier austênitique des cordons sains et profonds avec des zones affectêes thermiquement très réduites conduisant ainsi à des faibles déformations.

\section{REMFRCIEMENTS}

Ce travail a été réalisé en collaboration avec le centre de Soudage de Framatome (CATS) et 1'assistance technique de C. VIALLE (CALFETMAT). 